\title{
Rectangular Young tableaux and the Jacobi ensemble
}

\author{
Philippe Marchal $\|^{\| \dagger}$ \\ ${ }^{1}$ CNRS and Université Paris 13
}

\begin{abstract}
It has been shown by Pittel and Romik that the random surface associated with a large rectangular Young tableau converges to a deterministic limit. We study the fluctuations from this limit along the edges of the rectangle. We show that in the corner, these fluctuations are gaussian whereas, away from the corner and when the rectangle is a square, the fluctuations are given by the Tracy-Widom distribution. Our method is based on a connection with the Jacobi ensemble.

Résumé. Pittel et Romik ont établi l'existence d'une forme limite pour la surface aléatoire associée à un tableau de Young rectangulaire de grande taille. Nous étudions les fluctuations autour de cette limite le long du bord du rectangle. Nous montrons que ces fluctuations sont gaussiennes en les coins et, dans le cas où le rectangle est un carré, données par la loi de Tracy-Widom sur les bords. Notre méthode s'appuie sur un lien avec l'ensemble de Jacobi.
\end{abstract}

Keywords. Young tableau, Jacobi ensemble, Tracy-Widom distribution, arctic circle

\section{Statement of the results}

From a formal point of view, a rectangular Young tableau of size $(m, n)$ can be defined as an $m n$-tuple of integers $\left(X_{1,1}, \ldots X_{m, n}\right)$ satisfying, for all $i, j$,

$$
\begin{gathered}
X_{i, j}<\min \left(X_{i, j+1}, X_{i+1, j}\right) \\
\left\{X_{1,1}, \ldots X_{m, n}\right\}=\{1,2, \ldots, m n\}
\end{gathered}
$$

We denote by $\mathcal{X}_{m, n}$ the set of $m n$-tuples of this form.

Pittel and Romik [13] studied the limit shape of the surface associated with a large random rectangular Young tableau. Fix a real $t>0$ and consider a Young tableau of size $(n,\lfloor t n\rfloor)$, chosen uniformly at random. Then they proved that for all reals $(r, s) \in[0,1]^{2}, X_{\lfloor r n\rfloor,\lfloor s t n\rfloor} / t n^{2}$ converges in distribution as $n \rightarrow \infty$ to a deterministic quantity $g(r, s, t)$, which is the solution of a minimization problem. We want to study the fluctuations of this shape along the edges of the rectangle. First, we state a completely explicit result on the corner:

\footnotetext{
†Email: marchal@math.univ-paris13.fr. Partially supported by the projects "Alea Sorbonne" and "Combinatoire à Paris"

1365-8050 (C) 2016 Discrete Mathematics and Theoretical Computer Science (DMTCS), Nancy, France
} 
Theorem 1 Let $\left(X_{1,1}, \ldots, X_{m, n}\right)$ be a uniform random variable on $\mathcal{X}_{m, n}$. Then for $n \leq k \leq m n-m+1$,

$$
\mathbb{P}\left(X_{1, n}=k\right)=\frac{\left(\begin{array}{c}
k-1 \\
n-1
\end{array}\right)\left(\begin{array}{c}
m n-k \\
m-1
\end{array}\right)}{\left(\begin{array}{c}
m n \\
m+n-1
\end{array}\right)}
$$

Let us compare this result with the following model. Let $R$ be a rectangle of size $((m-1)(n-1), m+$ $n-1)$ on an integer lattice. Consider the set of paths going from the south-west corner to the north-east corner with only north and east steps. Choose such a path uniformly at random. Then it is an elementary exercise to check that the probability for the $n$-th north step to be the $k$-th step is equal to (1). However, we have not been able to find a combinatorial link between this simple model and the corner of a rectangular Young tableau. Also, note that the expected value of $X_{1, n}$ (and, more generally, of the north east corner of a random Young tableau with given skew shape) can be computed using Lemma 10 in [5], but the latter result does not give the exact distribution.

A consequence of Theorem 1 is the following (we use the notation $\stackrel{\text { law }}{\rightarrow}$ for the convergence in distribution):

Corollary 1 Fix some real $t \in \mathbb{R}_{>0}$. For every $n$, put $m_{n}=\lfloor$ tn $\rfloor$ and let $\left(X_{1,1}^{(n)}, \ldots, X_{m_{n}, n}^{(n)}\right)$ be a uniform random variable on $\mathcal{X}_{m_{n}, n}$. Then as $n \rightarrow \infty$,

$$
\frac{\sqrt{2}(1+t)\left(X_{1, n}^{(n)}-\mathbb{E} X_{1, n}^{(n)}\right)}{n^{3 / 2}} \stackrel{\text { law }}{\rightarrow} G
$$

where $G$ is a standard gaussian random variable.

Our second result only holds when the rectangle is a square and deals with the fluctuations on the edges.

Theorem 2 For each $n$, let $\left(X_{1,1}^{(n)}, \ldots, X_{n, n}^{(n)}\right)$ be a uniform random variable on $\mathcal{X}_{n, n}$. Then for every $t \in(0,1)$, as $n \rightarrow \infty$,

$$
\frac{r(t)\left(X_{1,\lfloor t n\rfloor}^{(n)}-\mathbb{E} X_{1,\lfloor t n\rfloor}^{(n)}\right)}{n^{4 / 3}} \stackrel{\text { law }}{\rightarrow} T W
$$

where $T W$ has the Tracy-Widom distribution and the function $r$ is defined by

$$
r(t)=\frac{2^{-1 / 3}(1+t)^{1 / 2}(1-t)^{1 / 6}}{t^{4 / 3}}
$$

Pittel-Romik's result tells us that $n^{-2} \mathbb{E} X_{\lfloor t n\rfloor, n}^{(n)}$ converges to the value of the limit shape at $(t, 1)$, namely $\left(1+\sqrt{2 t-t^{2}}\right) / 2$. Also note that $X_{1,\lfloor t n\rfloor}^{(n)}$ has an interpretation in terms of the longest increasing subsequence for a class of permutations that are called random extremal Erdós-Szekeres permutations in [13]. In particular, Theorem 2 solves the second open problem mentioned in [13].

Our method consists in studying Young tableaux in a slightly modified framework. As the asymptotic shape only makes sense when the "height" is renormalized, that is, the $X_{i, j}$ are divided by $m n$, it seems natural to work directly in a continuous framework. The formal setup is the following. 
Definition. For a pair of integers $(m, n)$, let $\mathcal{Y}_{m, n}$ be the set of $m n$-tuples $\left(Y_{1,1}, \ldots Y_{m, n}\right)$ of reals in $[0,1]$ satisfying, for all $i, j$,

$$
Y_{i, j}<\min \left(Y_{i, j+1}, Y_{i+1, j}\right)
$$

We want to study uniform random variables on $\mathcal{Y}_{m, n}$, which is called the order polytope of the rectangle viewed as a poset (see [3] for related questions).

Denote by $\Delta\left(x_{1}, \ldots x_{k}\right)$ the Vandermonde of the $k$-tuple $\left(x_{1}, \ldots x_{k}\right)$ :

$$
\Delta\left(x_{1}, \ldots x_{k}\right)=\prod_{1 \leq i<j \leq k}\left(x_{i}-x_{j}\right)
$$

When the rectangle is a square, we get:

Theorem 3 Let $n$ be a positive integer and $\left(Y_{1,1}, \ldots, Y_{n, n}\right)$ be a uniform random variable on the set $\mathcal{Y}_{n, n}$. Then for every $k \in[1, n]$, the $k$-tuple $\left(Y_{1, n-k+1}, Y_{2, n-k+2}, \ldots, Y_{k-1, n-1}, Y_{k, n}\right)$ has a marginal density proportional to

$$
\mathbf{1}_{\left\{x_{1} \leq x_{2} \ldots \leq x_{k}\right\}} \Delta\left(x_{1}, \ldots x_{k}\right)^{2} \prod_{i=1}^{k} x_{i}^{n-k}\left(1-x_{i}\right)^{n-k}
$$

For a general rectangle, the result reads:

Theorem 4 Let $m<n$ be two positive integers. Let $\left(Y_{1,1}, \ldots, Y_{m, n}\right)$ be a uniform random variable on the set $\mathcal{Y}_{m, n}$.

(i) Let $k \in[1, m]$ be an integer. Then the $k$-tuple $\left(Y_{1, n-k+1}, Y_{2, n-k+2}, \ldots, Y_{k-1, n-1}, Y_{k, n}\right)$ has a marginal density proportional to

$$
\mathbf{1}_{\left\{x_{1} \leq x_{2} \ldots \leq x_{k}\right\}} \Delta\left(x_{1}, \ldots x_{k}\right)^{2} \prod_{i=1}^{k} x_{i}^{n-k}\left(1-x_{i}\right)^{m-k}
$$

(ii) If $m<k \leq n$, then the $m$ - tuple $\left(Y_{1, n-k+1}, Y_{2, n-k+2}, \ldots, Y_{m-1, n-k+m-1}, Y_{m, n-k+m}\right)$ has a marginal density proportional to

$$
\mathbf{1}_{\left\{x_{1} \leq x_{2} \ldots \leq x_{k}\right\}} \Delta\left(x_{1}, \ldots x_{k}\right)^{2} \prod_{i=1}^{k} x_{i}^{n-k}\left(1-x_{i}\right)^{k-m}
$$

Of course, for a diagonal of the form $\left(Y_{m-k+1,1}, Y_{m-k+2,2}, \ldots, Y_{m, k}\right)$, we get a similar expression as in (i). The densities appearing in these two theorems belong to the general class called the Jacobi ensemble. This ensemble appears in various models, among others the MANOVA procedure in statistics [12], log-gas theory [8], Wishart matrices and random projections [6]. For a detailed account on random matrices, we refer to [1].

Using Theorems 3 and 4 together with known results on the Jacobi ensemble enables us to derive the results stated above. Moreover, the deterministic limit shape can also be recovered this way, see Section 4. An alternative form of Theorem 2 in the continuous setting is as follows: 
Corollary 2 For each integer $n$, let $\left(Y_{1,1}^{(n)}, \ldots, Y_{n, n}^{(n)}\right)$ be a uniform random variable on $\mathcal{Y}_{n, n}$. Then for $t \in(0,1)$, with the same function $r$ as in Theorem 2 , as $n \rightarrow \infty$,

$$
\frac{r(t)\left(Y_{1,\lfloor t n\rfloor}^{(n)}-\mathbb{E} Y_{1,\lfloor t n\rfloor}^{(n)}\right)}{n^{4 / 3}} \stackrel{\text { law }}{\rightarrow} T W
$$

where TW has the Tracy-Widom distribution.

Remark that $\mathcal{Y}_{n, n}$ is a convex subset (indeed, a compact polytope) of $\mathbb{R}^{n^{2}}$. From this point of view, Corollary 2 can be seen as a result on the projection of the uniform measure on a convex set in high dimension. This is reminiscent of the classical result saying that if $\left(X_{1}, \ldots X_{n}\right)$ is a random vector distributed according to the uniform measure on the euclidean $n$-dimensional sphere with radius $\sqrt{n}$, then $X_{1}$ is asymptotically gaussian.

The remainder of this note is organized as follows. We prove Theorems 3 and 4 in Section 2. Theorem 1 is derived in Section 3. Section 4 is devoted to the proofs of the asymptotic results. Some concluding remarks are made in Section 5

\section{Diagonals of continuous tableaux}

We prove here Theorem 3. The proof of Theorem 4 is similar and is omitted. The basic idea is to use a random generation algorithm filling the tableau diagonal by diagonal, using conditional densities.

We begin with a preliminary lemma. Let $n \geq 2$ and define by induction the following polynomials: $g_{1}(x)=1$ and for $i \leq n-1$,

$$
g_{i+1}\left(x_{1}, \ldots x_{i+1}\right)=\int_{x_{1}}^{x_{2}} d y_{1} \ldots \int_{x_{i}}^{x_{i+1}} d y_{n} g_{i}\left(y_{1}, \ldots, y_{i}\right)
$$

while for $i \in[n, 2 n-2]$,

$$
g_{i+1}\left(x_{1}, \ldots x_{2 n-i-1}\right)=\int_{0}^{x_{1}} d y_{1} \int_{x_{1}}^{x_{2}} d y_{2} \ldots \int_{x_{2 n-i-1}}^{1} d y_{2 n-i} g_{i}\left(y_{1}, \ldots, y_{2 n-i}\right)
$$

Lemma 1 (i) For every $i \in[1, n]$, there exists a constant $c_{i}$ such that

$$
g_{i}\left(x_{1}, \ldots x_{i}\right)=c_{i} \Delta\left(x_{1}, \ldots x_{i}\right)
$$

(ii) For $i \in[n, 2 n-1]$, there exists a constant $c_{i}$ such that

$$
g_{i}\left(x_{1}, \ldots x_{2 n-i}\right)=c_{i} \Delta\left(x_{1}, \ldots x_{2 n-i}\right) \prod_{j=1}^{2 n-i} x_{j}^{i-n}\left(1-x_{j}\right)^{i-n}
$$

\section{Proof}

An elementary proof of (i) can be found in Baryshnikov [2]. To deduce (ii), we proceed by induction. For $m \leq n$, define $K_{m}\left(\varepsilon, y_{1}, \ldots y_{n-m}\right)$ as the integral

$$
\int_{-\varepsilon}^{0} d r_{1} \ldots \int_{-m \varepsilon}^{-(m-1) \varepsilon} d r_{m} \int_{1}^{1+\varepsilon} d s_{1} \ldots \int_{1+(m-1) \varepsilon}^{1+m \varepsilon} d s_{m} \Delta\left(r_{1}, \ldots r_{m}, y_{1}, \ldots y_{n-m}, s_{1}, \ldots s_{m}\right)
$$


We want to evaluate

$$
I:=\int_{0}^{x_{1}} d y_{1} \ldots \int_{x_{n-m-1}}^{1} d y_{n-m} K_{m}\left(\varepsilon, y_{1}, \ldots y_{n-m}\right)
$$

On the one hand, using (i) easily gives

$$
I \sim \varepsilon^{-m(m+1)} C(m, n) \Delta\left(x_{1}, \ldots, x_{n-m-1}\right) \prod_{i=1}^{n-m-1} x_{i}^{m+1}\left(1-x_{i}\right)^{m+1}
$$

for some positive constant $C(m, n)$, where the equivalent is uniform over all $(n-m)$-tuples $\left(x_{1}, \ldots x_{n-m}\right)$ satisfying

$$
\sqrt{\varepsilon} \leq x_{1} \leq \ldots \leq x_{n-m} \leq 1-\sqrt{\varepsilon}
$$

On the other hand,

$$
\Delta\left(r_{1}, \ldots y_{1}, \ldots s_{m}\right) \sim \Delta\left(y_{1}, \ldots y_{n-m}\right) \Delta\left(r_{1}, \ldots s_{1}, \ldots s_{m}\right) \prod_{i=1}^{n-m} y_{i}^{m}\left(1-y_{i}\right)^{m}
$$

where the equivalent is uniform over all $(n+m)$-tuples $\left(r_{1}, \ldots y_{1}, \ldots s_{m}\right)$ satisfying

$$
\begin{gathered}
\sqrt{\varepsilon} \leq y_{1} \leq \ldots \leq y_{n-m} \leq 1-\sqrt{\varepsilon} \\
-\varepsilon<r_{1}<0, \ldots,-m \varepsilon<r_{m}<-(m-1) \varepsilon, 1<s_{1}<1+\varepsilon, \ldots
\end{gathered}
$$

As a consequence,

$$
K_{m}\left(\varepsilon, y_{1}, \ldots y_{n-m}\right) \sim C^{\prime}(m, n) \varepsilon^{m(m+1)} \Delta\left(y_{1}, \ldots, y_{n-m}\right) \prod_{i=1}^{n-m} y_{i}^{m}\left(1-y_{i}\right)^{m}
$$

where $C^{\prime}(m, n)$ is the constant such that

$$
\int_{-\varepsilon}^{0} d r_{1} \ldots \int_{1+(m-1) \varepsilon}^{1+m \varepsilon} d s_{m} \Delta\left(r_{1}, \ldots s_{1}, \ldots s_{m}\right) \sim C^{\prime}(m, n) \varepsilon^{m(m+1)}
$$

It follows that uniformly over all $(n-m)$-tuples $\left(x_{1}, \ldots x_{n-m}\right)$ satisfying (5),

$$
I \sim C^{\prime}(m, n) \varepsilon^{m(m+1)} \int_{0}^{x_{1}} d y_{1} \ldots \int_{x_{n-m-1}}^{1} d y_{n-m} \Delta\left(y_{1}, \ldots, y_{n-m}\right) \prod_{i=1}^{n-m} y_{i}^{m}\left(1-y_{i}\right)^{m}
$$

Comparing this estimate with (4) yields (ii).

We now describe an algorithm generating random elements in $\mathcal{Y}_{n, n}$. A similar algorithm can be used to generate random permutations with a prescribed profile of ascents and descents [11]. In the remainder of this section, $k$ and $n$ are the integers in the statement of Theorem 3. For $i \in[1, n]$, we denote

$$
D_{i}=\left(Y_{1, n-i+1}, Y_{2, n-i+2}, \ldots, Y_{i, n}\right)
$$


while for $i \in[n+1,2 n-1]$,

$$
D_{i}=\left(Y_{i-n+1,1}, Y_{i-n+2,2}, \ldots, Y_{n, 2 n-i}\right)
$$

If $\left(x_{1}, \ldots x_{j}\right)$ and $\left(y_{1}, \ldots y_{j+1}\right)$ are two sequences of reals, we say that they are interlacing in $[0,1]$ if $0 \leq y_{1} \leq x_{1} \leq y_{2} \ldots \leq x_{j} \leq y_{j+1} \leq 1$. We denote the event that this interlacing relation is satisfied by

$$
\operatorname{Inter}\left(\left(x_{1}, \ldots x_{j}\right),\left(y_{1}, \ldots y_{j+1}\right)\right)
$$

\section{Algorithm}

- Choose the diagonal $D_{k}$ at random according to the density

$$
\frac{\mathbf{1}_{\left\{0 \leq x_{1} \leq x_{2} \ldots \leq x_{k} \leq 1\right\}} g_{k}\left(x_{1}, \ldots x_{k}\right) g_{2 n-k}\left(x_{1}, \ldots x_{k}\right)}{Z_{k}}
$$

where

$$
Z_{k}=\int_{0}^{1} d x_{k} \int_{0}^{x_{k}} d x_{k-1} \ldots \int_{0}^{x_{2}} d x_{1} g_{k}\left(x_{1}, \ldots x_{k}\right) g_{2 n-k}\left(x_{1}, \ldots x_{k}\right)
$$

- By induction, for $i$ from $k$ down to 2 , conditional on $D_{i}$, choose $D_{i-1}$ according to the conditional density

$$
\frac{g_{i-1}\left(x_{1}, \ldots, x_{i-1}\right) \mathbf{1}_{\operatorname{Inter}\left(D_{i},\left(x_{1}, \ldots, x_{i-1}\right)\right)}}{g_{i}\left(D_{i}\right)}
$$

- By induction, for $i$ from $k$ to $n-1$, conditional on $D_{i}$, choose $D_{i+1}$ according to the conditional density

$$
\frac{g_{i+1}\left(x_{1}, \ldots, x_{i+1}\right) \mathbf{1}_{\operatorname{Inter}\left(D_{i},\left(x_{1}, \ldots, x_{i+1}\right)\right)}}{g_{i}\left(D_{i}\right)}
$$

- By induction, for $i$ from $n$ to $2 n-2$, conditional on $D_{i}$, choose $D_{i+1}$ according to the conditional density

$$
\frac{g_{i+1}\left(x_{1}, \ldots, x_{2 n-i-1}\right) \mathbf{1}_{\operatorname{Inter}\left(D_{i},\left(x_{1}, \ldots, x_{2 n-i-1}\right)\right)}}{g_{i}\left(D_{i}\right)}
$$

First, remark that the conditional densities used by the algorithm are indeed probability densities. That is, they are measurable, positive functions and their integral is 1 . The latter fact is easy to verify: for instance, by definition of $g_{i}$,

$$
g_{i}\left(D_{i}\right)=g_{i}\left(Y_{1}, \ldots Y_{i}\right)=\int_{Y_{1}}^{Y_{2}} d x_{1} \ldots \int_{Y_{i-1}}^{Y_{i}} d x_{i-1} g_{i-1}\left(x_{1}, \ldots, x_{i-1}\right)
$$

and thus, using $(6)$, we get

$$
\int_{[0,1]^{i-1}} d x_{1} \ldots d x_{i-1} \frac{g_{i-1}\left(x_{1}, \ldots, x_{i-1}\right) \mathbf{1}_{\operatorname{Inter}\left(D_{i},\left(x_{1}, \ldots, x_{i-1}\right)\right)}}{g_{i}\left(D_{i}\right)}=1
$$


We claim that the algorithm yields a random element of $\mathcal{Y}_{n, n}$ with the uniform measure. Indeed, by construction, the $n^{2}$-tuple generated by the algorithm has a density which is the product of the conditional densities of the diagonals $D_{1}, D_{2} \ldots D_{2 n-1}$. Hence this density is given by

$$
\frac{\mathbf{1}_{\left\{0 \leq x_{1} \leq \ldots \leq x_{k} \leq 1\right\}} g_{k}\left(D_{k}\right) g_{2 n-k}\left(D_{k}\right)}{Z_{k}} \prod_{i=2}^{k} \frac{g_{i-1}\left(D_{i-1}\right) \mathbf{1}_{\operatorname{Inter}\left(D_{i}, D_{i-1}\right)}}{g_{i}\left(D_{i}\right)} \prod_{i=k+1}^{2 n-1} \frac{g_{i}\left(D_{i}\right) \mathbf{1}_{\operatorname{Inter}\left(D_{i}, D_{i-1}\right)}}{g_{i-1}\left(D_{i-1}\right)}
$$

The expression above is a telescopic product and after simplification, we find that the density is constant on the set $\mathcal{Y}_{n, n}$. This proves our claim.

Finally, the density of $D_{k}$ is proportional to

$$
\mathbf{1}_{\left\{x_{1} \leq \ldots \leq x_{k}\right\}} g_{k}\left(x_{1}, \ldots x_{k}\right) g_{2 n-k}\left(x_{1}, \ldots x_{k}\right)=\mathbf{1}_{\left\{x_{1} \leq \ldots \leq x_{k}\right\}} \Delta\left(x_{1}, \ldots x_{k}\right)^{2} \prod_{i=1}^{k} x_{i}^{n-k}\left(1-x_{i}\right)^{n-k}
$$

according to Lemma 1 . This proves Theorem 3.

\section{The law of the corner}

One can relate the discrete and the continuous model of Young tableaux. To construct a continuous Young tableau $\left(Y_{i, j}\right)$ of size $(m, n)$ from a discrete Young tableau $\left(X_{i, j}\right)$ of the same size, proceed as follows:

- Let $\left(X_{i, j}\right)$ be a uniform random variable on $\mathcal{X}_{m, n}$.

- Let $\left(Z_{1} \leq \ldots \leq Z_{m n}\right)$ be the increasing reordering of $m n$ independent, uniform random variables on $[0,1]$, independent of $\left(X_{i, j}\right)$.

- For every pair $(i, j)$, let $k(i, j)$ be the integer satisfying $X_{i, j}=k(i, j)$. Then put $Y_{i, j}=Z_{k(i, j)}$.

Proposition 1 Consider the $(m n)$-tuple $\left(Y_{i, j}\right)$ constructed as above. Then

(i) $\left(Y_{i, j}\right)$ is distributed according to the uniform measure on $\mathcal{Y}_{m, n}$,

(ii) For every pair $(i, j)$,

$$
\mathbb{E}\left(Y_{i, j}-\frac{X_{i, j}}{m n+1}\right)^{2} \leq \frac{1}{m n+1}
$$

The proof of (i) is elementary and (ii) follows from a simple variance computation, using the fact that the density of $Z_{k}$ is

$$
h_{k}(x)=x^{k-1}(1-x)^{m n-k} \frac{(m n) !}{(k-1) !(m n-k) !}
$$

As a consequence of Proposition 1 , let $f_{i, j}$ be the marginal density of $Y_{i, j}$. For every $1 \leq k \leq m n$ put $p_{i, j}(k)=\mathbb{P}\left(X_{i, j}=k\right)$. Using Proposition 1 , we get that the density $f_{i, j}$ is equal to

$$
f_{i, j}(x)=\sum_{k=1}^{m n} p_{i, j}(k) h_{k}(x)
$$


where $h_{k}(x)$ is the density of $Z_{k}$. Thus

$$
f_{i, j}(x)=\sum_{k=1}^{m n} p_{i, j}(k) x^{k-1}(1-x)^{m n-k} \frac{(m n) !}{(k-1) !(m n-k) !}
$$

This way one can deduce the probabilities in the discrete model from the densities in the continuous model. For the case $i=1, j=n$, according to Theorem 4 (i),

$$
f_{1, n}(x)=x^{n-1}(1-x)^{m-1} \frac{(m+n-1) !}{(m-1) !(n-1) !}
$$

To obtain the desired decomposition, divide both sides of 7 by $(1-x)^{m n-1}$ and use the change of variables $y=x /(1-x)$ to get

$$
\frac{(m+n-1) !}{(m-1) !(n-1) !} y^{n-1}(1+y)^{m n-n-m+1}=\sum_{k=1}^{m n} p_{1, n}(k) y^{k-1} \frac{(m n) !}{(k-1) !(m n-k) !}
$$

Identifying the coefficient of $y^{k-1}$ for each $k$ yields Theorem 1 .

\section{Asymptotic results}

\subsection{Proof of Theorem 2}

For every $n$, let $\left(Y_{1,1}^{(n)}, \ldots, Y_{n, n}^{(n)}\right)$ be distributed according to the uniform measure on $\mathcal{Y}_{n, n}$. As a consequence of Collins' results on the "soft edge" of the Jacobi ensemble [6], we get the following. For every $t \in(0,1)$, there exists a family $\left(s_{n, m}(t)\right)$ and a constant $r(t)$ given by (2) such that, for every $k$, as $n$ goes to infinity,

$$
r(t) n^{2 / 3}\left(Y_{1,\lfloor t n\rfloor}^{(n)}-s_{n, 1}(t), \ldots, Y_{k,\lfloor t n\rfloor+k-1}^{(n)}-s_{n, k}(t)\right)
$$

converges in distribution to a $k$-tuple $\left(A_{1}, \ldots, A_{k}\right)$ which is the truncation of a random point process $\left(A_{1}>A_{2}>\ldots>A_{k}>\ldots\right)$ distributed according to the Airy ensemble. Moreover, the estimate (ii) of Proposition 1 entails that $(8)$ and

$$
r(t) n^{2 / 3}\left(\frac{X_{1,\lfloor t n\rfloor}^{(n)}}{n^{2}}-s_{n, 1}(t), \ldots, \frac{X_{k,\lfloor t n\rfloor+k-1}^{(n)}}{n^{2}}-s_{n, k}(t)\right)
$$

have the same limit. This yields Theorem 2. Remark that Theorem 2 is a specialization to the case $k=1$ but that we have in fact a multidimensional convergence:

Theorem 5 With the same assumptions and notation as in Theorem 2 , for every $k \geq 1$, the $k$-tuple

$$
r(t) n^{2 / 3}\left(\frac{X_{1,\lfloor t n\rfloor}^{(n)}-\mathbb{E} X_{1,\lfloor t n\rfloor}^{(n)}}{n^{2}}, \ldots, \frac{X_{k,\lfloor t n\rfloor+k-1}^{(n)}-\mathbb{E} X_{k,\lfloor t n\rfloor+k-1}^{(n)}}{n^{2}}\right)
$$

converges in distribution towards the largest $k$ values of the Airy process. 
In fact, [6] gives a general result which applies for every rectangle. More precisely, for all $m, n, k$, the diagonal $\left(Y_{k, n}, \ldots, Y_{1, n-k-1}\right)$ of $\mathcal{Y}_{m, n}$ is a determinantal point process for which [6] provides estimates both on the edge and in the bulk of the spectrum. However, the convergence towards the Airy ensemble is only proven in the square case. The extension to the rectangular case would require some concentration inequalities that do not seem to be available yet.

\subsection{Proof of Corollary 1}

To prove Corollary 1, remark again that Proposition 1 (ii) enables us to deduce the limit law of $X_{1, n}^{(n)}$ from the limit law of $Y_{1, n}^{(n)}$. The density of $Y_{1, n}^{(n)}$ is $x^{n-1}(1-x)^{m_{n}-1}$. Put $r=1 /(1+t)$ and $T_{n}=$ $\sqrt{n-1}\left(Y_{1, n}^{(n)}-r\right)$. Then the density of $T_{n}$ has the form

$$
c_{n}\left(1+\frac{x}{r \sqrt{n-1}}\right)^{n-1}\left(1-\frac{x}{(1-r) \sqrt{n-1}}\right)^{\lfloor t n\rfloor-1}
$$

for some constant $c_{n}$. Asymptotic estimations easily yield Corollary 1 .

\subsection{The deterministic limit shape}

We focus here on the square case but the case of a rectangle can be dealt with similarly. Fix a real $t \in(0,1)$, let $m(t)=\lfloor t n\rfloor$ and consider the diagonal

$$
\left(D_{1}^{(t)}, D_{2}^{(t)}, \ldots D_{m(t)}^{(t)}\right):=\left(Y_{1, n-m(t)+1}, Y_{2, n-m(t)+2}, \ldots, Y_{m(t), n}\right)
$$

Consider the empirical measure

$$
\mu_{n}(t)=\frac{1}{m(t)} \sum_{i=1}^{m(t)} \delta_{D_{i}^{(t)}}
$$

General results on Jacobi ensembles apply in this case and we get that $\mu_{n}(t)$ converges in distribution, as $n$ goes to infinity, to the deterministic probability measure with density

$$
f_{t}(x):=\frac{\sqrt{\left(\lambda_{+}(t)-x\right)\left(x-\lambda_{-}(t)\right)}}{\pi x(1-x)} \mathbf{1}_{\left\{x \in\left[\lambda_{-}(t), \lambda_{+}(t)\right]\right\}}
$$

where

$$
\lambda_{ \pm}(t)=\frac{1 \pm \sqrt{t(2-t)}}{2}
$$

See for instance the first proposition in [7]. Reformulating this result, we get the following. Let $r, s \in$ $[0,1]^{2}, r \geq s$ and put $t=1-s+r$. Then as $n$ tends to infinity, $X_{\lfloor r n\rfloor,\lfloor s n\rfloor}$ converges in law to the Dirac point mass $\delta_{g(r, s)}$ where

$$
g(r, s)=F_{t}^{-1}(s / t)
$$

the function $F_{t}^{-1}$ being the inverse of the function

$$
F_{t}(x)=\int_{\lambda_{-}(t)}^{x} f_{t}(y) d y
$$


and $f_{t}$ being given by $(9)$ and $(10)$. The function $g$ is an alternative fomulation of the limit shape found by Pittel and Romik.

Remark however that this result is weaker than Pittel-Romik's, since it only gives the convergence along a diagonal, whereas Pittel and Romik show a uniform convergence on the whole rectangle. In fact, a major weakness of our method is that it only allows us to work on a single diagonal, and not on several diagonals simultaneously.

\section{Concluding remarks}

Consider the case when the rectangle is a square. It would be interesting to study the transition between the deterministic regime of $X_{n, n}$ and the fluctuations of order $n^{4 / 3}$ for $X_{\lfloor t n\rfloor, n}$, as well as the transition between the fluctuations of order $n^{4 / 3}$ for $X_{\lfloor t n\rfloor, n}$ and the fluctuations of order $n^{3 / 2}$ for $X_{1, n}$. A natural conjecture is the following:

Conjecture 1 Let $\left(a_{n}\right)$ be a nondecreasing sequence with $a_{n} \rightarrow \infty$ as $n \rightarrow \infty$. Then, up to a multiplicative constant,

$$
\begin{gathered}
\frac{a_{n}^{1 / 6}\left(X_{\left\lfloor a_{n}\right\rfloor, n}^{(n)}-\mathbb{E} X_{\left\lfloor a_{n}\right\rfloor, n}^{(n)}\right)}{n^{3 / 2}} \stackrel{\text { law }}{\rightarrow} T W \\
\frac{X_{\left\lfloor a_{n}\right\rfloor, 1}^{(n)}-\mathbb{E} X_{\left\lfloor a_{n}\right\rfloor, 1}^{(n)}}{a_{n}^{4 / 3}} \stackrel{\text { law }}{\rightarrow} T W
\end{gathered}
$$

where $T W$ has the Tracy-Widom distribution.

This conjecture follows from the asymptotics of the function $r$ given by (2) when $t \rightarrow 0$ and $t \rightarrow 1$. Alternatively, the first part of the conjecture can be related to the random matrix model known as the GUE. For a fixed $k$, define a family of variables $\left(T_{i, k}^{(n)}, 1 \leq i \leq k\right)$ by the formula

$$
Y_{i, n-k+i}=\frac{1}{2}\left(1-\frac{T_{i, k}^{(n)}}{\sqrt{n}}\right)
$$

Then it follows immediately from Theorem 3 that, as $n$ goes to infinity, the renormalized diagonal $\left(T_{i, k}^{(n)}, \ldots T_{k, k}^{(n)}\right)$ has a limit density proportional to

$$
\mathbf{1}_{\left\{x_{1} \leq x_{2} \ldots \leq x_{k}\right\}} \Delta\left(x_{1}, \ldots x_{k}\right)^{2} \prod_{i=1}^{k} \exp \left(-x_{i}^{2} / 2\right)
$$

This is the density of the eigenvalues of a random $(k, k)$ matrix from the GUE, and classical results [1] naturally lead to the first part of the conjecture. Proving it would involve an exchange of limits, which does not seem to have been achieved in the literature so far.

There is a link between rectangular Young tableaux and a particle system known as the TASEP, see [14]. In this view, a phenomenon of arctic circle arises when the rectangle is a square. In the general case, the arctic curve is no longer a circle but it is still algebraic. To compute an equation of this curve, one has 
to determine the parameters of the Jacobi ensemble associated with the rectangle using Theorem 4, and then compute the values $\lambda_{ \pm}$for this Jacobi ensemble as in [7].

The horizontal strips of the TASEP diagram where this arctic curve appears correspond to diagonals of the Young tableau. Moreover, the places of the vertical steps inside a horizontal strip correspond to the integers in the diagonal of the Young tableau, and Theorems 3 and 4 tell us that these vertical steps are asymptotically distributed like the eigenvalues of a Jacobi ensemble. A similar result has been established by Johansson and Nordenstam for domino tilings of the Aztec diamond [10], where the Jacobi ensemble is replaced by the GUE.

In the case of a GUE of size $n$, the fluctuations of the eigenvalues in the bulk are of order $\sqrt{\log n / n}$ and are asymptotically gaussian [9]. It is not clear whether the same behaviour occurs in our context.

A model of corners of Jacobi ensembles was studied recently by Borodin and Gorin [4], who showed a convergence to the gaussian free field. Their model is slightly different from ours, but it would be interesting to know whether their results could be transposed in our case.

Finally, the method used here can be applied to generate at random a standard filling of a general polyomino: compute the conditional densities of the diagonals, which will be polynomials given by multiple integrals, and then use a generating algorithm as in Section 2. Of course, the problem is that for a general polyomino, the corresponding polynomials will not have a simple form as for rectangles.

\section{Acknowledgements}

I thank Florent Benaych, Cédric Boutillier, Benoit Collins and Alain Rouault, as well as the referees for useful references.

\section{References}

[1] Anderson, G., Guionnet, A., Zeitouni, O., An introduction to random matrices. Cambridge Studies in Advanced Mathematics, 118 Cambridge University Press, Cambridge, 2010

[2] Baryshnikov, Y., GUEs and queues. Probab. Theory Related Fields 119 (2001), no. 2, 256-274.

[3] Baryshnikov, Y., Romik, D., Enumeration formulas for Young tableaux in a diagonal strip, Israel J. Math. 178 (2010), 157-186.

[4] Borodin, A., Gorin, G., General beta Jacobi corners process and the Gaussian Free Field, Communications on Pure and Applied Mathematics 68 (2015), 1774-1844.

[5] Chan, M., Martin, A., Pflueger, N., Teixidor i Bigas, M., Genera of Brill-Noether curves and staircase paths in Young tableaux, preprint, arXiv:1506.00516v1.

[6] Collins, B., Product of random projections, Jacobi ensembles and universality problems arising from free probability. Probab. Theory Related Fields 133 (2005), no. 3, 315-344.

[7] Dumitriu, I., Paquette, E., Global fluctuations for linear statistics of $\beta$-Jacobi ensembles. Random Matrices Theory Appl.1 (2012), no. 4

[8] Forrester, P. J., Log-gases and random matrices. London Mathematical Society Monographs Series, 34 Princeton University Press, Princeton, NJ, 2010. 
[9] Gustavsson, J., Gaussian fluctuations of eigenvalues in the GUE. Ann. I. H. Poincaré (2005) 151178.

[10] Johansson, K.; Nordenstam, E., Eigenvalues of GUE minors. Electron. J. Probab. 11 (2006), no. $50,1342-1371$.

[11] Marchal, P., Permutations with a prescribed descent set. Preprint

[12] Muirhead, R. J., Aspects of multivariate statistical theory. Wiley Series in Probability and Mathematical Statistics. John Wiley and Sons, Inc., New York, 1982.

[13] Pittel, B., Romik, D., Limit shapes for random square Young tableaux. Adv. in Appl. Math. 38 (2007), no. 2, 164-209.

[14] Romik, D., Arctic circles, domino tilings and square Young tableaux. Ann. Probab. 40 (2012), no. 2, 611-647. 